\title{
Pulmonary Embolism in a Critically III Infant with Univentricular Parallel Circulation
}

\author{
Kazuhiro Shoya ${ }^{1}$ Jun Maeda ${ }^{2}$ Hiroki Nagamine ${ }^{2}$ Akihiro Shimotakahara ${ }^{3}$ Yukihiro Yoshimura ${ }^{4}$ \\ Osamu Saito ${ }^{1}$ \\ ${ }^{1}$ Department of Critical Care and Emergency Medicine, Tokyo \\ Metropolitan Children's Medical Center, Tokyo, Japan \\ ${ }^{2}$ Department of Cardiology, Tokyo Metropolitan Children's Medical \\ Center, Tokyo, Japan \\ ${ }^{3}$ Department of Surgery, Tokyo Metropolitan Children's Medical \\ Center, Tokyo, Japan \\ ${ }^{4}$ Department of Cardiovascular Surgery, Tokyo Metropolitan \\ Children's Medical Center, Tokyo, Japan \\ J Child Sci 2021;11:e212-e215.

\begin{abstract}
Address for correspondence Kazuhiro Shoya, MD, Sakakibara Heart Institute, 3-16-1 Asahicho, Fuchu, Tokyo 183-0003, Japan (e-mail: kaz_shoya@me.com).
\end{abstract}

\begin{abstract}
Keywords

- pulmonary embolism

- decreased pulmonary blood flow

- hypoplastic left heart syndrome

- cyanotic congenital heart disease

- univentricular parallel circulation
\end{abstract}

A 3-month-old infant patient with hypoplastic left heart syndrome diagnosed in the prenatal period required long-term intensive care for refractory chylothorax and chylous ascites after undergoing bilateral pulmonary artery banding at age 6 days. Weaning from mechanical ventilation was difficult due to massive edema, and a central venous catheter was required because enteral feeding was hampered by the refractory chyle leakage, for which surgery was ineffective. On the evening after central venous catheter replacement was performed, his respiratory condition suddenly deteriorated; cardiac ultrasound revealed that the left pulmonary arterial blood flow had decreased, and enhanced computed tomography demonstrated a left pulmonary embolism (PE), which was identified as the cause of a sudden decrease in the pulmonary blood flow. The patient died due to refractory septic shock at age 5 months. PEs in children, especially patients with cyanotic congenital heart disease, are difficult to diagnose because of their atypical presentation. Current diagnostic criteria are based on adult guidelines, and a few factors in the criteria, such as tachycardia and hypoxia, are difficult to apply for pediatric cyanotic patients with intracardiac or extracardiac rightto-left shunt. In fact, those criteria have lower specificities in children. In conclusion, the present case suggested that a sudden decrease in the pulmonary blood flow can aid the physicians in diagnosing PE in patients with cyanotic congenital heart disease. We need more pediatric cases and evidence of PE in children to make a PE guideline, which is specific to pediatric patients including cyanotic congenital heart disease.

\section{Introduction}

Pulmonary embolisms (PEs) are rarer in children than in adults, with previous studies reporting an estimated incidence of $0.14-0.9: 100,000$ in the pediatric population and

received

March 17, 2021

accepted after revision June 14, 2021
DOI https://doi.org/ 10.1055/s-0041-1733873. ISSN 2474-5871.
8.6-57:100,000 even among hospitalized children. ${ }^{1-4}$ Diagnosing PEs on the basis of their clinical presentations is difficult because of their potential nonspecificity and the inability of young children to describe their symptoms accurately. ${ }^{5}$ Another reason for the difficulty is that

\section{(c) 2021. The Author(s).}

This is an open access article published by Thieme under the terms of the Creative Commons Attribution License, permitting unrestricted use, distribution, and reproduction so long as the original work is properly cited. (https://creativecommons.org/licenses/by/4.0/) Georg Thieme Verlag KG, Rüdigerstraße 14, 70469 Stuttgart, Germany 
symptoms related to low cardiac output are infrequent in this population due to intracardiac or extracardiac right-to-left shunt. PE is highly associated with deep vein thrombosis (DVT), and the most salient predisposing factors in children are the presence of a central venous catheter (CVC), infection, and congenital heart disease (CHD). ${ }^{5}$

We herein report an atypical presentation of $\mathrm{PE}$ in a patient with hypoplastic left heart syndrome (HLHS). To our knowledge, a sudden decrease in the pulmonary blood flow can be a valuable clinical clue to diagnosing PE in patients with cyanotic CHD.

\section{Case Report}

A male infant was born at 37 weeks' gestation by cesarean section with a birth weight of 2,348 g to a 36-year-old G1P0 with atypical psychosis and gestational hypertension. HLHS (aortic atresia, mitral stenosis) was diagnosed during the fetal stage by echocardiogram. He was admitted to the neonatal intensive care unit (ICU) where he received a lipo-prostaglandin $\mathrm{E}_{1}$ infusion to maintain systemic blood flow through the ductus arteriosus. Within 1 day, the patient was intubated for low systemic blood flow with increased pulmonary blood flow, but his circulation remained unstable. Therefore, he was transferred to the pediatric ICU at age 3 days and started on inhaled $\mathrm{N}_{2}$. His $\mathrm{PaCO}_{2}$ was maintained between 50 and $60 \mathrm{~mm} \mathrm{Hg}$ to reduce the pulmonary blood flow. At age 6 days, he received bilateral pulmonary arterial banding (bil.PAB) for the increased pulmonary blood flow. Bil.PAB with an 8.5- $\mathrm{mm}$ circumference was performed for each pulmonary artery but required adjustment at age 13 days because of a continued increase in the pulmonary blood flow. A large amount of pleural effusion and ascites had drained after the first operation, and it was diagnosed with chyle leakage by increased white blood cell with a lymphocyte-predominant pattern in drainage. The chyle leakage was caused by elevated central venous pressure. Moreover, the refractory chylothorax and chylous ascites required continued CVC placement and plasma protein supplementation, including albumin, globulin, and coagulation factors. As a result, the patient experienced two episodes of persistent bacteremia. The refractory chyle leakage continued to be treated using drugs as well as lymphangiography but was unable to be suppressed. Options for CVC placement gradually dwindled because almost all the remaining central veins except the right internal jugular vein (RIJV) were obstructed by thrombosis due to long-term CVC placement despite prophylactically anticoagulation by low-dose unfractionated heparin (UFH) infusion. Fortunately, the right upper central vein from the RIJV to the superior vena cava was patent although venous thrombosis was detected in the superior vena cava in the early stage of the patient's clinical course.

At age 3 months, the patient underwent CVC replacement. On the night following the procedure, his respiratory status

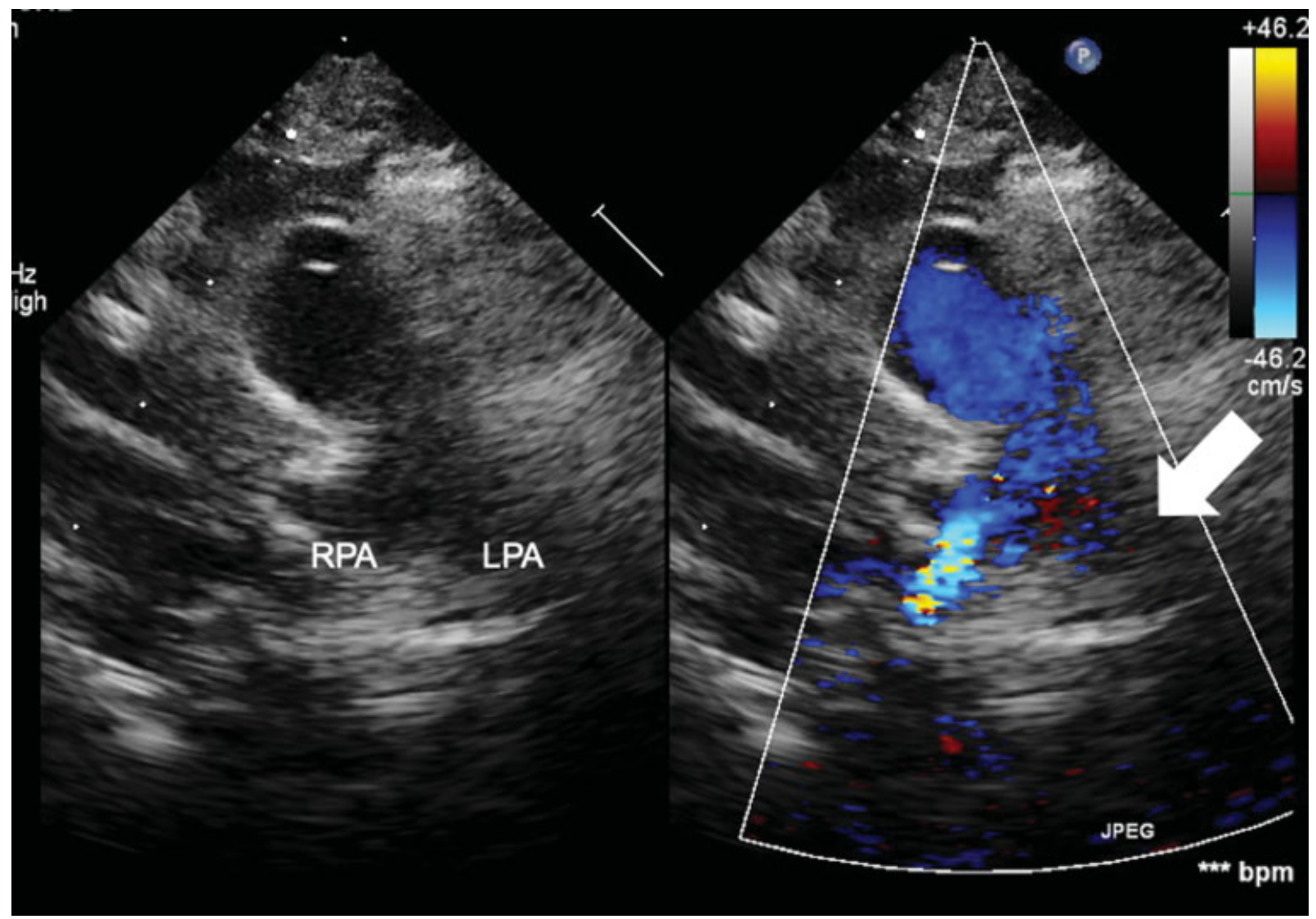

Fig. 1 Cardiac ultrasound at the diagnosis of the pulmonary embolism showing a decrease in the left pulmonary artery flow (white arrow). LPA, left pulmonary artery; RPA, right pulmonary artery. 


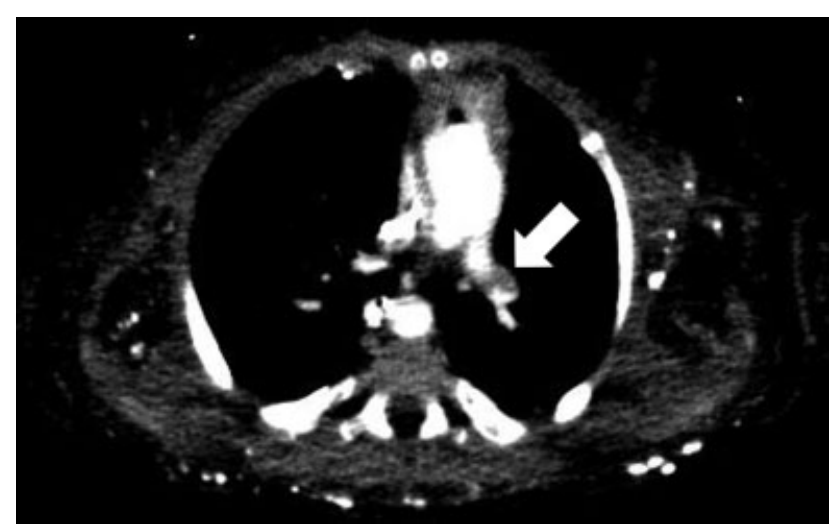

Fig. 2 Enhanced CT at the diagnosis of the pulmonary embolism showing a thrombus (white arrow) in the main left pulmonary artery. $\mathrm{CT}$, computed tomography.

suddenly deteriorated; his $\mathrm{SpO}_{2}$ fell from 75 to $68 \%$, and end tidal $\mathrm{CO}_{2}$ and $\mathrm{PaCO}_{2}$ rose from 36 to $53 \mathrm{~mm} \mathrm{Hg}$ and 52 to $66 \mathrm{~mm} \mathrm{Hg}$, respectively, within a few minutes. Nevertheless, the tidal volume and blood pressure were stable, and a chest $\mathrm{X}$-ray revealed no findings which might explain the sudden change in his respiratory status. Laboratory tests showed no specific findings. Echocardiogram, performed to evaluate the pulmonary blood flow on the suspicion that a change in hemodynamics caused the sudden deterioration of the respiratory status, found decreased left pulmonary arterial blood flow compared with that before the onset (-Fig. 1). Left PE was diagnosed on the basis of enhanced computed tomography (CT) findings ( - Fig. 2). Despite the presence of a thrombus, the D-dimer level remained stable at $0.9 \mu \mathrm{g} / \mathrm{mL}$. Catheter placement for the embolism was unable to be performed because the other central veins were no longer patent. UFH was increased to retard progression of the PE. The decreased pulmonary blood flow gradually improved with echocardiographic finding, but follow-up enhanced CT was not performed because it is difficult to transport due to the systemic deterioration with refractory sepsis. Finally, the patient died from refractory septic shock at age 5 months.

\section{Discussion}

Generally, PE in the pediatric population occurs in patients with CHD or an infection, with most cases being associated with CVC use. ${ }^{5}$ The incidence of venous pulmonary thromboembolisms has been steadily increasing in children as a consequence of longer survival times in critically ill children, as well as increased CVC use. ${ }^{6-8}$ Several studies reported that thrombotic events occurred at a rate of 33 to $64 \%$ in children with a CVC and 89 to $94 \%$ in neonates alone. ${ }^{1-3}$

In the present case, the patient had several PE risk factors, such as CHD, long-term use of CVC, a past history of blood stream infections, and DVT related to CVC use.

Typical symptoms of PE are signs of right heart failure, such as tachycardia, tachypnea, and biventricular edema. Patients with PE usually experience shortness of breath, pleuritic chest pain, hemoptysis, cough, and syncope. ${ }^{9,10}$ However, PE among children may occur without this classic symptomatology. Additionally, some symptoms may be related to underlying medical conditions, such as CHD, that often coincide with, and predispose the patient to, PE, thus masking the diagnosis. ${ }^{9}$

The present patient had extracardiac right-to-left shunt via the ductus arteriosus and so did not demonstrate symptoms of low cardiac output. Instead, his pulmonary-tosystemic flow ratio (Qp/Qs) changed dynamically because the left pulmonary artery was almost completely obstructed by thrombosis. Decreased Qp/Qs due to the PE led to the sudden deterioration of his respiratory status.

Due to the nonspecificity of the clinical presentation of PE, diagnostic imaging is necessary for a definitive diagnosis. Even in the absence of abnormalities in the initial laboratory findings, blood gas, and electrocardiogram, further investigation is called for if PE is suspected. The initial tests for PE should be performed while the imaging modalities are being prepared. ${ }^{5}$

Previous studies have made specific testing recommendations for diagnosing PE. D-dimer, a biomarker useful in detecting an existing thrombosis, is one such example. However, a previous study enrolling 50 children with PE and 25 PE-negative control patients showed that it had low utility for diagnosing PE. ${ }^{11}$ Another study demonstrated that the D-dimer level was normal in $40 \%$ of children with PE. ${ }^{12}$ In adults, Wells' criteria and Pulmonary Embolism Rule-out Criteria rules are known as common PE algorithms. However, they should be used cautiously in children because these criteria have lower specificities in pediatric patients. ${ }^{13}$ Furthermore, they include a few factors included in these algorithms, such as tachycardia and hypoxia are difficult to apply for patients with cyanotic CHD. Echocardiogram may be useful, especially in the ICU setting, ${ }^{14,15}$ because it enables the patient's condition before and after PE onset to be compared. Radiologic evaluations, such as enhanced CT, ventilation-perfusion scan, pulmonary angiography, and magnetic resonance imaging, can also be useful for diagnosing PE. ${ }^{16}$ In particular, enhanced CT is highly recommended for young patients in the ICU or emergency setting because it is noninvasive and rapid. ${ }^{17}$ CT has improved, and multidetector CT pulmonary angiography has been recently regarded as the routine modality for detection of $\mathrm{PE}$ in both adults and children. ${ }^{18,19}$ In the present case, the Ddimer level had a poor predictive value for thrombosis. Although the patient's symptoms were atypical, their sudden onset was a clue to diagnosing thrombosis. In the present case, echocardiogram demonstrated high utility in the ICU setting and in predicting the PE. However, a definitive diagnosis of PE requires a highly specific radiologic examination, such as enhanced CT.

No specific treatment algorithms are currently available for managing PE in children. ${ }^{16}$ Previous studies have presented various management options, such as anticoagulant therapy, thrombolysis, catheter intervention, inferior vena cava filter, and thrombectomy. Anticoagulant therapy includes UFH, lowmolecular-weight heparin, and vitamin K antagonist administration. $^{5}$ The only remaining treatment choice in the present patient, in view of his condition, was UFH. 
The choice of treatments depends on the individual condition of the patient. Current therapeutic recommendations are based on the findings of adult clinical trials and a few pediatric studies enrolling a small number of subjects. Therefore, we should conduct clinical trials in childhood PE to make PE guidelines for children.

\section{Conclusion}

Our findings suggested that sudden-onset hypoxia and hypercapnia should raise the suspicion of decreased pulmonary blood flow due to PE. Once PE is suspected, a diagnostic workup and radiologic evaluations should be performed. Once the diagnosis is made, treatment should be started without delay.

PE in children is rare and difficult to diagnose. Furthermore, PE in patients with cyanotic CHD often has an atypical presentation and is even more difficult to diagnose because intracardiac or extracardiac right-to-left shunt maintains the systemic blood flow.

We need more pediatric cases and evidence of PE to make a PE guideline which is specific to pediatric patients including cyanotic CHD.

\section{Authors' Contributions}

K.S. drafted the manuscript; J.M. and O.S. revised the manuscript and made scientific contributions; J.M., H.N., A.S., Y.Y., and O.S. managed the patient; all the authors read and approved the final manuscript.

\section{Conflict of Interest}

None declared.

\section{Acknowledgments}

We thank James Robert Valera for his assistance with editing the manuscript.

\section{References}

1 Andrew M, David M, Adams M, et al. Venous thromboembolic complications (VTE) in children: first analyses of the Canadian Registry of VTE. Blood 1994;83(05):1251-1257

2 Biss TT, Brandão LR, Kahr WH, Chan AK, Williams S. Clinical features and outcome of pulmonary embolism in children. $\mathrm{Br} \mathrm{J}$ Haematol 2008;142(05):808-818
3 van Ommen CH, Heijboer H, Büller HR, Hirasing RA, Heijmans HS, Peters M. Venous thromboembolism in childhood: a prospective two-year registry in The Netherlands. J Pediatr 2001;139(05): 676-681

4 Stein PD, Kayali F, Olson RE. Incidence of venous thromboembolism in infants and children: data from the National Hospital Discharge Survey. J Pediatr 2004;145(04):563-565

5 Dijk FN, Curtin J, Lord D, Fitzgerald DA. Pulmonary embolism in children. Paediatr Respir Rev 2012;13(02):112-122

6 Byard RW. Fatal embolic events in childhood. J Forensic Leg Med 2013;20(01):1-5

7 Patocka C, Nemeth J. Pulmonary embolism in pediatrics. J Emerg Med 2012;42(01):105-116

8 Male C, Julian JA, Massicotte P, Gent M, Mitchell LPROTEKT Study Group. Significant association with location of central venous line placement and risk of venous thrombosis in children. Thromb Haemost 2005;94(03):516-521

9 Brandão LR, Labarque V, Diab Y, Williams S, Manson DE. Pulmonary embolism in children. Semin Thromb Hemost 2011;37(07): 772-785

10 Bernstein D, Coupey S, Schonberg SK. Pulmonary embolism in adolescents. Am J Dis Child 1986;140(07):667-671

11 Biss TT, Brandão LR, Kahr WHA, Chan AK, Williams S. Clinical probability score and D-dimer estimation lack utility in the diagnosis of childhood pulmonary embolism. J Thromb Haemost 2009;7(10):1633-1638

12 Rajpurkar M, Warrier I, Chitlur M, et al. Pulmonary embolismexperience at a single children's hospital. Thromb Res 2007;119 (06):699-703

13 Hennelly KE, Baskin MN, Monuteuax MC, et al. Detection of pulmonary embolism in high-risk children. J Pediatr 2016; 178:214-218.e3

14 Ramiz S, Rajpurkar M. Pulmonary embolism in children. Pediatr Clin North Am 2018;65(03):495-507

15 Rajpurkar M, Williams S, Goldenberg NA, et al. Results of a multinational survey of diagnostic and management practices of thromboembolic pulmonary embolism in children. Thromb Res 2019;183:98-105

16 Zaidi AU, Hutchins KK, Rajpurkar M. Pulmonary embolism in children. Front Pediatr 2017;5:170

17 Henzler T, Barraza JM Jr, Nance JW Jr, et al. CT imaging of acute pulmonary embolism. J Cardiovasc Comput Tomogr 2011;5(01): 3-11

18 Lee EY, Tse SKS, Zurakowski D, et al. Children suspected of having pulmonary embolism: multidetector CT pulmonary angiography-thromboembolic risk factors and implications for appropriate use. Radiology 2012;262(01):242-251

19 Tang CX, Schoepf UJ, Chowdhury SM, Fox MA, Zhang LJ, Lu GM. Multidetector computed tomography pulmonary angiography in childhood acute pulmonary embolism. Pediatr Radiol 2015;45 (10):1431-1439 